\title{
A Combinatorial Proof of the Log-concavity of a famous sequence counting permutations
}

\author{
Miklós Bóna * \\ Submitted: Nov 24, 2004; Accepted: Jan 11, 2005; Published: Jan 24, 2005 \\ Mathematics Subject Classifications: 05A05, 05A15
}

To Richard Stanley, who introduced me to the area of log-concave sequences.

\begin{abstract}
We provide a combinatorial proof for the fact that for any fixed $n$, the sequence $\{i(n, k)\}_{0 \leq k \leq\left(\begin{array}{c}n \\ 2\end{array}\right)}$ of the numbers of permutations of length $n$ having $k$ inversions is log-concave.
\end{abstract}

\section{Introduction}

Let $p=p_{1} p_{2} \cdots p_{n}$ be a permutation of length $n$, or, in what follows, an $n$-permutation. An inversion of $p$ is a pair $(i, j)$ of indices so that $i<j$, but $p_{i}>p_{j}$. The enumeration of $n$-permutations according to their number $i(p)$ of inversions, and the study of numbers $i(n, k)$ of $n$-permutations having $k$ inversions, is a classic area of combinatorics. The best-known result is the following [4].

Theorem 1.1 Let $n \geq 2$. Then we have

$$
\sum_{p \in S_{n}} x^{i(p)}=\sum_{k=0}^{\left(\begin{array}{c}
n \\
2
\end{array}\right)} i(n, k) x^{k}=(1+x)\left(1+x+x^{2}\right) \cdots\left(1+x+x^{2}+\cdots+x^{n-1}\right) .
$$

Another classic result [3] is that the numbers $i(n, k)$ also count $n$-permutations having major index $k$. Details about this result, and other related results can be found in [1].

${ }^{*}$ University of Florida, Gainesville FL 32611-8105. Partially supported by an NSA Young Investigator Award. Email: bona@math.ufl.edu. 
A somewhat less explored property of the numbers $i(n, k)$ is log-concavity. The sequence $\left(a_{k}\right)_{0 \leq k \leq m}$ is called log-concave if $a_{k} a_{k+2} \leq a_{k+1}^{2}$ for all $k$. See [5] for a classic survey of log-concave sequences, and see [2] for an update on that survey. A polynomial is called log-concave if its coefficients form a log-concave sequence. It is a classic result (see for instance [1] for a proof) that the product of log-concave polynomials is log-concave. Therefore, Theorem 1.1 immediately implies that the polynomial $\sum_{k=0}^{\left(\begin{array}{c}n \\ 2\end{array}\right)} i(n, k) x^{k}$ is $\log$ concave, that is, the sequence $i(n, 0), i(n, 1), \cdots, i\left(n,\left(\begin{array}{l}n \\ 2\end{array}\right)\right)$ is log-concave. We could not find any previous proof of this fact that does not use generating functions. In this paper, we will provide such a proof. It is also the first non-generating function proof we know of in which a sequence whose length is quadratic in terms of the length of the input objects is shown to be log-concave.

\section{The proof of our claim}

\subsection{The outline of the proof}

It is easy to see that the sequence $\left(a_{k}\right)_{0 \leq k \leq m}$ is log-concave if and only if $a_{k} a_{l} \leq a_{k+1} a_{l-1}$ for all $k \leq l-2$. One implication is trivial, and the other becomes obvious if we note that $\log$-concavity is equivalent to the sequence $a_{k+1} / a_{k}$ being weakly decreasing.

Let $p$ be an $n$-permutation, and set $p=p_{1} p_{2} \cdots p_{n}$. Define $I_{n, k}$ to be the set of all $n$-permutations with exactly $k$ inversions. When there is no danger of confusion about what $n$ is, we will just write $I_{k}$ instead of $I_{n, k}$.

The structure of our proof will be as follows. We want to prove the following theorem.

Theorem 2.1 For all integers $n, k$ and $l$ satisfying $0 \leq k \leq l-2 \leq\left(\begin{array}{l}n \\ 2\end{array}\right)-2$, there exists an injection $f_{n, k, l}: I_{k} \times I_{l} \rightarrow I_{k+1} \times I_{l+1}$.

Theorem 2.1 is clearly equivalent to what we want to prove. We will prove our claim by induction on $n$. That is, first, we will construct the injections $f_{n, k, l}$ for the smallest meaningful value of $n$, which is $n=3$. Then, in the induction step, we will use the assumption that the maps $f_{n-1, k, l}$ exist for all allowed values of $k$ and $l$ to create the maps $f_{n, k, k+2}$. We will not create the maps $f_{n, k, l}$ for $k<l-2$, but we do not have to, since the existence of the maps $f_{n, k, k+2}$ in itself implies the log-concavity of the sequence $\{i(n, k)\}_{0 \leq k \leq\left(\begin{array}{l}n \\ 2\end{array}\right)}$, and therefore, it implies the existence of the maps $f_{n, k, l}$ for $k<l-2$. That will complete the induction step of our proof.

\subsection{The details of the proof}

It is time that we carried out the strategy to prove Theorem 2.1 that we discussed in the previous subsection. 
The smallest value of $n$ for which the domains of the maps $f_{n, k, l}$ are not all empty is $n=3$. In this case, $f_{n, k, l}$ is defined for the $(k, l)$-pairs $(0,2),(0,3)$ and $(1,3)$. In those cases, we define $f_{3,0,2}(123,231)=(213,132), f_{3,0,2}(123,312)=(213,213)$, and $f_{3,0,3}(123,321)=$ $(213,231)$, as well as $f_{3,1,3}(132,321)=(231,231)$, and $f_{3,1,3}(213,321)=(312,231)$. It will soon become obvious why we define $f$ this way.

Now let $n \geq 4$, and assume we have defined $f_{n-1, k, l}$ for all allowed values of $k$ and $l$.

Let $(p, q) \in I_{k} \times I_{k+2}$, with $p=p_{1} p_{2} \cdots p_{n}$ and $q=q_{1} q_{2} \cdots q_{n}$. Proceed as follows.

(Rule 1) If $p_{1}<n$ and $q_{1}>1$, increase $p_{1}$ by one, and decrease the entry of $p$ that was one larger than $p_{1}$ by one. Let the obtained permutation be $p^{\prime}$. Similarly, decrease $q_{1}$ by 1 , and increase the entry of $q$ that was one larger than $q_{1}$ by 1 . Let the obtained permutation be $q^{\prime}$. Set $f_{n, k, k+2}(p, q)=\left(p^{\prime}, q^{\prime}\right)$.

Note that $p^{\prime}$ starts with an entry larger than 1 , and $q^{\prime}$ starts with an entry less than $n$.

Example 2.2 If $p=2134$ and $q=3142$, then we have $f_{4,1,3}(p, q)=(3124,2143)$.

(Rule 2) If $p_{1}=n$, or $q_{1}=1$, then remove these entries, to get the permutations $p *$ and $q *$. (After natural relabeling, these are both permutations of length $n-1$.) Because of the extreme values of at least one of the omitted elements, we have $i(q *)-i(p *) \geq$ $i(q)-i(p)=2$. Therefore, there exist positive integers $r$ and $s$, with $r \leq s-2$, so that $(p *, q *)$ is in the domain of $f_{n-1, r, s}$.

Take $f_{n-1, r, s}(p *, q *)=(\bar{p}, \bar{q}) \in I(n-1, r+1) \times I(n-1, s-1)$. Now prepend $\bar{p}$ by $p_{1}$, and prepend $\bar{q}$ by $q_{1}$. In both cases, entries larger than or equal to the prepended entry have to be increased by 1 . Call this new pair of $n$-permutations $\left(p_{1} \bar{p}, q_{1} \bar{q}\right)$. Finally, set $f_{n, k, k+2}(p, q)=\left(q_{1} \bar{q}, p_{1} \bar{p}\right)$. We point out that we swapped $p$ and $q$.

Note that either $q_{1} \bar{q}$ starts in 1 or $p_{1} \bar{p}$ starts in $n$.

Example 2.3 If $p=1324$ and $q=1432$, then we have $(p *, q *)=(213,321)$, therefore, recalling that we have already defined $f_{3,1,3}$ for 3-permutations, $f_{3,1,3}(p *, q *)=$ $(\bar{p}, \bar{q})=(312,231)$. Reinserting the removed first entries, we get $\left(p_{1} \bar{p}, q_{1} \bar{q}\right)=$ $(1423,1342)$. Finally, after swapping the two permutations of the last pair, we get $f_{4,1,3}(p, q)=(1342,1423)$.

Lemma 2.4 The map $f_{n, k, k+2}: I_{k} \times I_{k+2} \rightarrow I_{k+1} \times I_{k+1}$ is an injection.

Proof: First, it is clear that $f_{n, k, k+2}$ maps into $I_{k+1} \times I_{k+1}$ since both rules increase the number of inversions of the first permutation by one, and decrease the number of inversions of the second permutation by one.

Now we prove that $f_{n, k, k+2}$ is one-to-one. We achieve this by induction on $n$, the initial case of $n=3$ being obvious. Assume now that the statement is true for $n-1$. 
Let $(t, u) \in I_{k+1} \times I_{k+1}$, with $t=t_{1} t_{2} \cdots t_{n}$, and $u=u_{1} u_{2} \cdots u_{n}$. We show that $(t, u)$ can have at most one preimage under $f_{n, k, k+2}$. There are two cases.

1. If $t_{1}>1$ and $u_{n}<n$, then $(t, u)$ could only be obtained as a result of applying $f_{n, k, k+2}$ if Rule 1 was used. In that case, we have $f_{n, k, k+2}^{-1}(t, u)=\left(\left(t_{1}-1\right) t_{2} \cdots t_{n},\left(u_{1}+\right.\right.$ 1) $\left.u_{2} \cdots u_{n}\right)$.

2. If $t_{1}=1$, or $u_{1}=n$, then $(t, u)$ could only be obtained as a result of applying $f_{n, k, k+2}$ if Rule 2 was used. In that case, to get the preimage of $(t, u)$, we need to remove the first entry of $t$ and the first entry of $u$, swap the permutations, and find the preimage of the resulting pair $(\bar{u}, \bar{t})$ under the appropriate map $f_{n-1, r, s}$.

However, the preimage of $(\bar{u}, \bar{t})$ under $f_{n-1, r, s}$ is unique by the induction hypothesis, therefore so is $f_{n, k, k+2}^{-1}(t, u)$.

This completes our proof. $\diamond$

Consequently, the sequence $\{i(n, k)\}_{0 \leq k \leq\left(\begin{array}{c}n \\ 2\end{array}\right)}$ is log-concave, and the injections $f_{n, k, l}$ exist for all values $k$ and $l$ satisfying $0 \leq k \leq l-2 \leq\left(\begin{array}{l}n \\ 2\end{array}\right)-2$.

\section{Acknowledgement}

I am grateful to the anonymous referee for a careful reading of the manuscript.

\section{References}

[1] M. Bóna, Combinatorics of Permutations, CRC Press, 2004.

[2] F. Brenti, Log-concave and unimodal sequences in algebra, combinatorics, and geometry: an update, Jerusalem Combinatorics '93, Contemp. Math. 178 (1994), Amer. Math. Soc., Providence, RI, 71-89.

[3] P. A. MacMahon, The indices of permutations, and the derivation therefrom of functions of a single variable associated with the permutations of any assemblage of objects, Amer. J. Math. 35 (1913), 281-322.

[4] G. Rodrigues, Note sur les inversions, ou dérangements produits dans les permutations, J. Math. Pures Appl. 4 (1839), 236-240.

[5] R. Stanley, Log-concave and unimodal sequences in algebra, combinatorics, and geometry. Ann. New York Acad. Sci. 576 (1989), 500-535. 